\title{
Design of one non-linear adaptive control system and study of its tracking
}

\author{
Samiran Maiti ${ }^{1}$, Achintya Das ${ }^{2}$ \\ ${ }^{1}$ Department of ECE, Birbhum Institute of Engineering and Technology, Suri, Birbhum, West Bengal, India \\ ${ }^{2}$ Department of Electronics and Communication Engineering, Kalyani Govt. Engineering College, \\ Kalyani, Nadia, India
}

\begin{tabular}{l} 
Article Info \\
\hline Article history: \\
Received Jul 16, 2018 \\
Revised Oct 11, 2018 \\
Accepted Oct 25, 2018 \\
\hline
\end{tabular}

Keywords:

Adaption mechanism

Lyapunov method

Model reference adaptive

controller (MRAC)

Nonlinear plant

\section{Corresponding Author:}

Samiran Maiti,

Department of ECE,

Birbhum Institute of Engineering and Technology,

Suri, Birbhum, West Bengal, Pin-731101, India.

Email: samiran.cemk@gmail.com

\begin{abstract}
In this paper, an adaptive control method is proposed for a category of nonlinear systems. As the to begin with step in versatile controller plan, we select the control law which containing variable parameters. At that point select an adaption law for adjusting those parameters. We analyze the convergence properties and system stability using Lyapunov theory. The viability of the proposed approaches is appeared by implies of recreation on MATLAB
\end{abstract} All rights reserved.

\section{INTRODUCTION}

A system having uncertainty in process dynamics and external disturbances is difficult to control. Customary input controllers $[1,2]$ attempt to diminish the effects of inner and outside unsettling influences up to certain degree and they ordinarily perform palatably for a specific working condition. So, the necessity of a controller with a wide range of operating condition checking the unsettling influence and varying process dynamics causes the development of adaptive controller [3,4]. Nonlinear tracking control is one of the most important issues in control engineering and its applications [5]. Numerous down to earth building applications stand up to with nonlinear dynamics, in which frequently involve uncertainties in system parameters and parameter variations and/or disturbance. To overcome the over issue, various noteworthy control plans have been broadly pulled in a impressive sum of intrigued in most recent a few decades, such as adaptive control, fuzzy logic control, feedback linearization control, neural networks control, sliding mode control [6].

In the present work an MRAC is developed for single variable nonlinear plants, which can be applied to time invariant and time-varying plants without any modifications. In this method the differences between the coefficients that are determined by the plant parameters, in the differential equations describing the actual plant and the model plant are estimated. The nonlinearity can be due to inherent nonlinearity of the parameters and the time-varyingness can arise due to several factors such as inherent property of the parameters, wear and tear, changes in the operational conditions etc. This type of uncertainty is known as parametric or structured uncertainty. The estimates are then used to update the model reference adaptive 
controller parameters. Lyapunov's direct method is then used to obtain bounded signals when the controller is in use.

\section{MODEL REFERENCE ADAPTIVE CONTROLLER}

Figure 1 shows the MRAC. It is composed of four parts: (1) Plant: it is assumed that plant have known structure, even though the parameters are not known. (2) Reference Model: A reference model is used to specify the ideal response of the adaptive control system to the external command. The choice of the reference model has to fulfill two requirements.

- It must satisfy the specifications which are used for performance in the control tasks.

- The reference model should reach the ideally behavior for the adaptive control system.

(3) Controller: It is generally standardized by a number of adjustable parameters. That implies there exist different sets of controller parameter values for which the desired control task is achievable. Usually in terms of the adjustable parameters the control law is linear. Here only one control parameter $\theta$ is taken. The value of $\theta$ dependent on adaptation gain. Parameter Adjustment block: parameters in the control law are adjusted here. Adaptation law finds the parameters in such a way that the response of the plant will follow the reference model. It is designed to achieve the stability of the control system as well as for reduction of tracking error to zero.

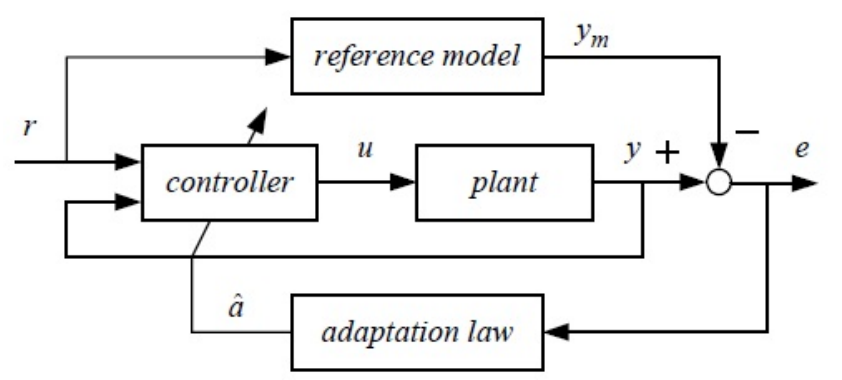

Figure 1. Model reference adaptive control system

\section{Estimation Of Parameters}

In this work we consider nonlinear first order plant described by the differential (1)

$$
(d y / d t)=-a_{p} y-c_{p} f(y)+b_{p} u
$$

Where $f$ is any known non linear function. The nonlinear in these dynamics is characterized by its linear parameterization in terms of the unknown constant c. The model plant is given by (2)

$$
\left(d y_{m} / d t\right)=-a_{m} y_{m}+b_{m} r
$$

Now we use the control law (3)

$$
u=\hat{a}_{y} y+\hat{a}_{f} f(y)+\hat{a}_{r} r
$$

where the second term in (2) is introduced with the intention of adaptively cancelling the nonlinear term. $\hat{a}_{r}$ and $\hat{a}_{\boldsymbol{y}}$ are variable feedback gain. With the control law, the closed loop dynamics is (4)

$$
\begin{aligned}
& (\mathbf{d y} / \mathbf{d t})=-\mathbf{a}_{\mathbf{p}} \mathbf{y}-\mathbf{c}_{\mathbf{p}} \mathbf{f}(\mathbf{y})+\mathbf{b}_{\mathbf{p}}\left(\hat{a}_{y} y+\hat{a}_{f} f(y)+\hat{a}_{r} r\right) \\
& (\mathbf{d y} / \mathbf{d t})=-\left(\mathbf{a}_{\mathbf{p}}-\mathbf{b}_{\mathbf{p}} \hat{a}_{y}\right) \mathrm{y}-\left(\mathbf{c}_{\mathbf{p}}-\mathbf{b}_{\mathbf{p}} \hat{a}_{f}\right) \mathbf{f}(\mathbf{y})+\mathbf{b}_{\mathbf{p}} \hat{a}_{r \mathbf{r}}
\end{aligned}
$$

Substituting this control law into the dynamics (1) and subtracting the resulting equation by (2), we obtain the error dynamics. 


$$
\dot{e}=\frac{1}{k_{r}^{*}} M\left(\tilde{a}_{y} y+\tilde{a}_{f} f(y)+\tilde{a}_{r} r\right)
$$

Where the parameter error ${ }^{\widetilde{a}_{f}}$ is defined as

$$
\tilde{a}_{f}=\hat{a}_{f}-\frac{c_{p}}{b_{p}}
$$

The adaption laws are:

$$
\begin{aligned}
& \dot{\hat{a}}_{y}=-\operatorname{sgn}\left(b_{p}\right) \gamma e y \\
& \dot{\hat{a}}_{f}=-\operatorname{sgn}\left(b_{n}\right) \gamma e f \\
& \dot{\hat{a}}_{r}=-\operatorname{sgn}\left(b_{p}\right) \gamma e r
\end{aligned}
$$

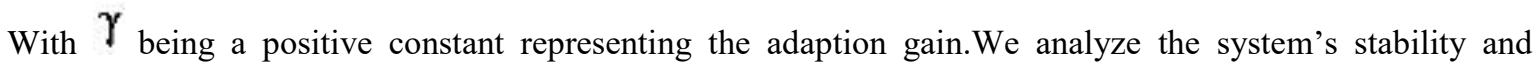
convergence behavior using the Lyapunov function.

\section{SIMULATION RESULTS} representation is

Here we have examine the proposed method by using a first order non-linear plant whose

$$
(\mathrm{dy} / \mathrm{dt})=\mathrm{y}+\mathrm{y}^{2}+2.9 \mathrm{u}
$$

The plant parameters are:

$$
\mathrm{a}_{\mathrm{p}}=-1, \mathrm{bp}=2.9
$$

We consider the reference model parameters are:

$$
\mathrm{a}_{\mathrm{m}}=4, \mathrm{bm}=4 \text {. }
$$

Let adaption gain $\boldsymbol{\gamma}_{=2} 2$ and $\mathrm{y}(0)=\mathrm{ym}(0)=0$

For reference signal $r(t)=4$, the tracking performance is shown in Figure 2. The parameter estimator is shown in Figure 3.

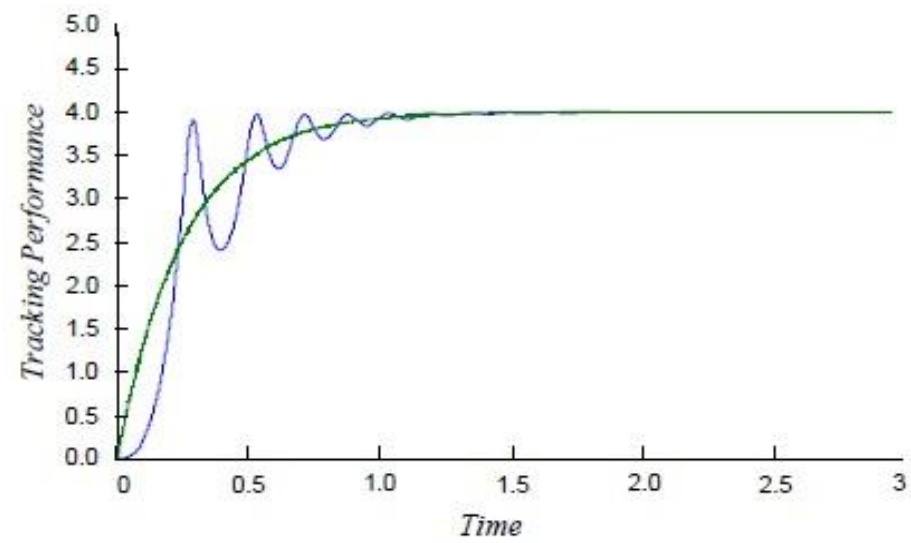

Figure 2. The tracking performance, for reference signal $r(t)=4$ 


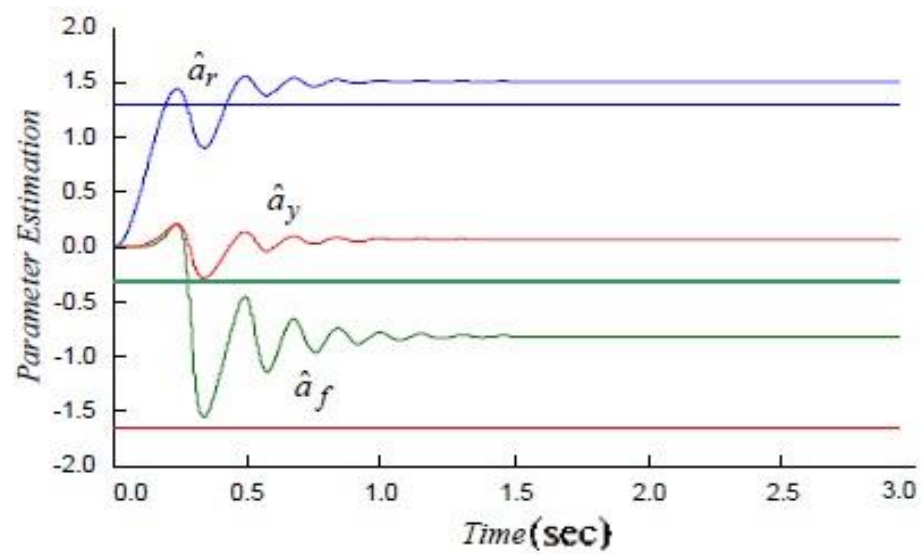

Figure 3. The parameter estimator, for reference signal $r(t)=4$

For reference signal $r(t)=4 \sin (3 t)$, the tracking performance is shown in Figure 4 . The parameter estimator is shown in Figure 5.

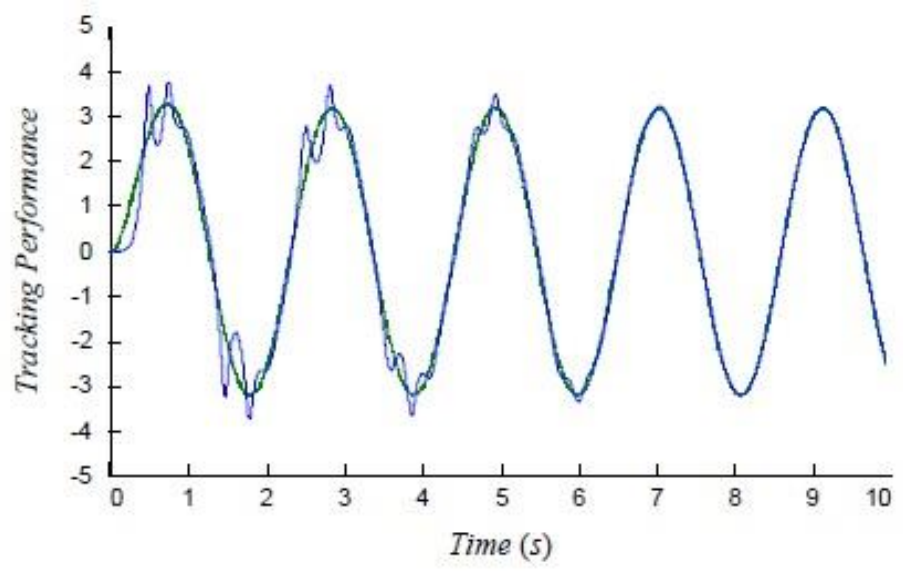

Figure 4. The tracking performance, for reference signal $r(t)=4 \sin (3 t)$

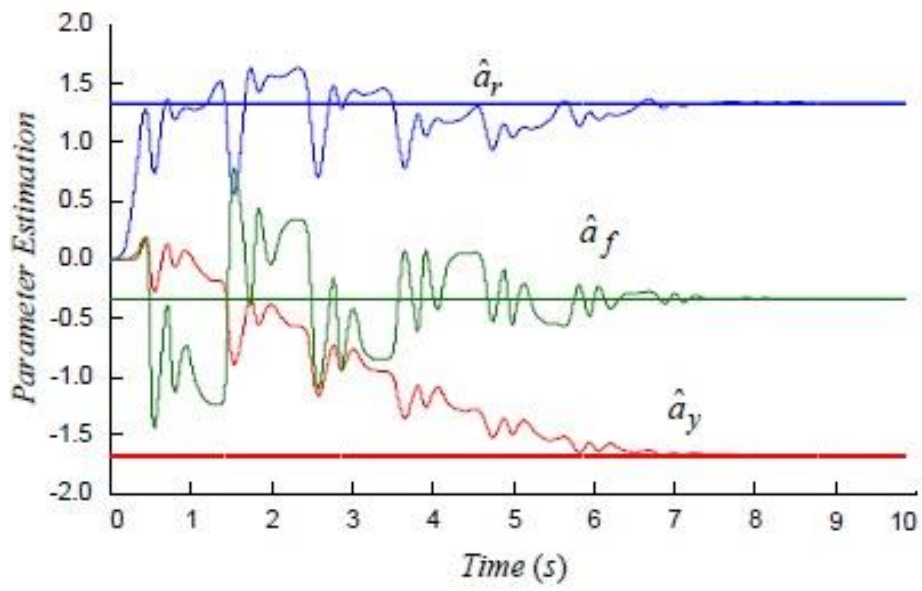

Figure 5. The parameter estimator, for reference signal $r(t)=4 \sin (3 t)$ 


\section{CONCLUSION}

In this paper, we have considered a Model Reference Adaptive Control, which is design according to Lyapunov stability, applicable to a class of uncertain systems nonlinearities. Under upper norm bounded conditions for system uncertainties, it has been proved that the proposed model reference adaptive control scheme is proposed with an excellent yield following property with regard to framework vulnerabilities. A few numerical recreation comes about have too been given to illustrate the validity of the proposed control strategy.

\section{REFERENCES}

[1] Astrom, K. J., and Wittenmark, B., “Adaptive Control," (2. Ed).

[2] Krsti C, M., Kanellakopoulos, I. and Kokotovi C, P. V., "Nonlinear and Adaptive Control Design,” Wiley, 1995.

[3] Miyasato, Y. and Hanba, S., "Adaptive control for nonlinear systems with unknown degrees And uncertain relative degrees," Preprints of 1 1th IFAC Symposium on System Identification, pp.943-948, Fukuoka, Japan, 1997.

[4] P. A. Ioannou and J. Sun, "Robust Adaptive Control," PTR Prentice-Hall, 1996.

[5] Y. Miyasato, "On nonlinear adaptive control systems independent of the degree of the process," Korean Automatic Control Conference, pp.740-745, 1988.

[6] A. K. Chakrabarty and Prof. S. Bhattacharya, "Adaptive control of a plant with time-varying structured uncertainties-a new approach," Journal of Institution of Engineers (India), vol.90, pp.3-8, March 2010. 